\title{
DeepBeam - A Machine Learning Framework For Tuning The Primary Electron Beam of The PRIMO Monte Carlo Software
}

\section{Zbisław Tabor ( $\sim$ tabor.zbislaw@gmail.com )}

AGH University of Science and Technology: Akademia Gorniczo-Hutnicza imienia Stanislawa Staszica w Krakowie https://orcid.org/0000-0002-9688-9718

\section{Damian Kabat}

Maria Skłodowska Curie Memorial Cancer Centre and Institute of Oncology Krakow Department of Medical Physics: Centrum Onkologii-Instytut im Marii Sklodowskiej-Curie Oddzial w Krakowie Zaklad Fizyki Medycznej

\section{Michael Waligórski}

Tadeusz Kosciuszko Cracow University of Technology: Politechnika Krakowska im Tadeusza Kosciuszki

\section{Research}

Keywords: Machine learning, deep learning, Monte Carlo, beam simulation, quality assurance, QA, quality control QC, PCA-principal component analysis, support vector regression

Posted Date: April 26th, 2021

DOl: https://doi.org/10.21203/rs.3.rs-425712/v1

License: (c) (1) This work is licensed under a Creative Commons Attribution 4.0 International License. Read Full License

Version of Record: A version of this preprint was published at Radiation Oncology on June 29th, 2021. See the published version at https://doi.org/10.1186/s13014-021-01847-w. 
2 DeepBeam - a machine learning framework for tuning the primary

${ }^{(1)}$ Zbisław Tabor, ${ }^{(2)}$ Damian Kabat, ${ }^{(3)}$ Michael Waligórski

7

(1) AGH University of Science and Technology,

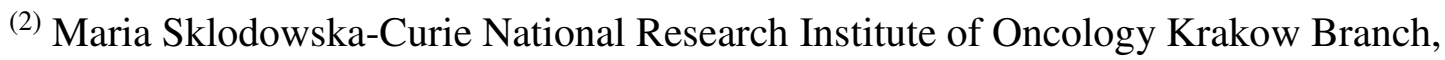
Garncarska 11, 31-115 Krakow, Poland, z5kabat@cyfronet.pl

(3) Cracow University of Technology, Podchorążych 1, 30-084 Kraków, Poland, z5waligo@cyf-kr.edu.pl

16 Author to whom correspondence should be addressed: Zbisław Tabor,

17 tabor.zbislaw@gmail.com

\section{Abstract}

\section{Background}

22 Any Monte Carlo simulation of dose delivery using medical accelerator-generated megavolt

23 photon beams begins by simulating electrons of the primary electron beam interacting with a

24 target. Because the electron beam characteristics of any single accelerator are unique and

25 generally unknown, an appropriate model of an electron beam must be assumed before MC 
26 simulations can be run. The purpose of the present study is to develop a flexible framework

27 with suitable regression models for estimating parameters of the model of primary electron

28 beam in simulators of medical linear accelerators, basing on real reference dose profiles

29 measured in a water phantom.

\section{Methods}

31 All simulations were run using PRIMO MC simulator. Two regression models for estimating

32 the parameters of the simulated primary electron beam, both based on machine learning, were

33 developed. The first model applies Principal Component Analysis to measured dose profiles

34 in order to extract principal features of the shapes of the these profiles. The PCA-obtained

35 features are then used by Support Vector Regressors to estimate the parameters of the model

36 of the electron beam. The second model, based on deep learning, consists of a set of encoders

37 processing measured dose profiles, followed by a sequence of fully connected layers acting 38 together, which solve the regression problem of estimating values of the electron beam

39 parameters directly from the measured dose profiles. Results of the regression are then used to

40 reconstruct the dose profiles, basing on the PCA model. Agreement between the measured and

41 reconstructed profiles can be further improved by an optimization procedure resulting in the

42 final estimates of the parameters of the model of the primary electron beam. These final 43 estimates are then used to determine dose profiles in MC simulations.

\section{Results}

45 Analysed were a set of actually measured (real) dose profiles of $6 \mathrm{MV}$ beams from a real 46 Varian $2300 \mathrm{C} / \mathrm{D}$ accelerator, a set of simulated training profiles, and a separate set of 47 simulated testing profiles, both generated for a range of parameters of the primary electron 48 beam of the Varian 2300 C/D PRIMO simulator. Application of the two-stage procedure based 49 on regression followed by reconstruction-based minimization of the difference between 50 measured (real) and reconstructed profiles resulted in achieving consistent estimates of 
51 electron beam parameters and in a very good agreement between the measured and simulated

52 photon beam profiles.

\section{Conclusions}

54 The proposed framework is a readily applicable and customizable tool which may be applied

55 in tuning virtual primary electron beams of Monte Carlo simulators of linear accelerators. The

56 codes, training and test data, together with some trained models and readout procedures, are

57 freely available at the site: https://github.com/taborzbislaw/DeepBeam.

60 Key words: Machine learning, deep learning, Monte Carlo, beam simulation, quality

61 assurance, QA, quality control QC, PCA-principal component analysis, support vector

62 regression.

63

64

65

66

67

68

69

70

71

72

73

74

75 


\section{Introduction}

77 External photon beam therapy (EBT) is nowadays the most common cancer radiotherapy

78 modality. The key factors which determine the success of EBT are correct and accurate

79 treatment therapy planning and quality assurance procedures prior to delivery of the

80 therapeutic dose. Designing a therapy plan is a multi-dimensional optimization problem. The

81 therapy planning system (TPS) designs therapy plan to match the therapy goals within

82 specified clinical tolerances. Only after assuring that these goals are met, may the quality

83 assurance procedures of dose delivery be implemented and the patient treated.

84 The dose calculation algorithms are the true engines of all TPS systems. The first 85 generation Pencil Beam Convolution (PBC) algorithm calculates the delivered dose by 86 convoluting the ionizing radiation field intensity (or fluence) with pencil beam kernels and by

87 applying subsequent corrections for patient tissue heterogeneity, as based on CT data [1]. The next generation Anisotropic Analytical Algorithm (AAA) is a semi-analytical model which

89 also uses pencil beams. The AAA pencil beams are determined using the photon beam data of

90 a linac. Heterogeneity corrections in the AAA algorithm also account for scattered radiation

91 [2]. The ACUROSE XB (AXB) algorithm solves deterministically a coupled system of linear

92 Boltzmann transport equations to model the transport of ionizing radiation through a 93 homogeneous medium, subsequently applying heterogeneity corrections and explicitly

94 modelling the interaction of radiation with matter, basing on patient CT data [3]. As all the 95 aforementioned algorithms are only approximations, they must be thoroughly tested against a 96 reference gold standard.

97 In modern radiotherapy, Monte Carlo (MC)-calculated dose distributions are currently 98 the primary reference tool for verifying other dose-calculation methods, or for benchmarking

99 commercial dose calculation algorithms. Ultimately, MC modelling is expected to replace the 100 current approximate algorithms in therapy planning [4]. It is generally accepted that Monte 
101 Carlo simulation of dose delivery is the most reliable and accurate method of representing the

102 real aspects of calculating dose distributions [5]. This is because MC modelling incorporates

103 all aspects of photon and electron transport in generally heterogeneous media. However, while

104 being potentially very accurate and extremely valuable in gaining thorough understanding of 105 all phenomena related to dose deposition in various media, it is also a very challenging task

106 [4]. This is due not only to the high computational effort required by MC modelling, but also

107 because of the tuning process which must be carefully implemented to match MC-calculated 108 doses and doses measured under controlled conditions. Tuninig of a MC simulator of a 109 medical accelerator involves three basic steps, namely geometry tuning, virtual electron beam 110 tuning, and dose unit calibration.

111 Any MC simulation of dose delivery by megavolt photon beams from a medical linear

112 accelerator commences by simulating the primary beam of high-energy electrons which leave 113 the acceleration tube of the linac with a well-specified energy of several MeV impinging a

114 carefully designed tungsten cone target to generate megavolt photons via bremsstrahlung. The

115 spatial distribution of dose delivered by the thus generated photon beam to a water phantom 116 or to the patient's tumour volume, crucially depends on the characteristics of the primary 117 electron beam. To MC-model the details of photon beam production and of its distribution, 118 scattering and shaping by various collimators and instruments located in the head of the linac, 119 their exact geometry configuration, material composition and construction of the head of a 120 commercially produced medical linear accelerator must be known in great detail. Given the 121 high precision of manufacture and assembly of all mechanical parts of a modern linac, its 122 head geometry and construction details are not expected to significantly vary within a 123 production line of accelerators of a given type from a given manufacturer. This considerably 124 facilitates implementation of geometry considerations in MC calculations when simulating a 125 given model of a commercially produced linear accelerator. Yet, the primary electron beam 
126 characteristics of any individual accelerator are unique. Moreover, the characteristics of their

127 primary electron beams may vary not only between linacs of the same type, but may also vary

128 in time within the same accelerator, due to ageing effects [6-8]. Unfortunately, the geometry

129 and spectra of the primary electron beam are neither known exactly nor easily measurable,

130 except by quite specialized equipment, which is not readily available in a typical clinical

131 radiotherapy environment $[9,10]$.

132 For this reason, to run MC simulations, an appropriate model of the electron beam

133 must be designed, which includes a model of its electron energy spectrum and model of its

134 spatial distribution. Typically, no less than four parameters are needed to characterise a MC-

135 simulated electron beam of a medical linear accelerator - namely the mean energy of

136 electrons in the beam, the full width at half-maximum of the electron energy spectrum in the

137 beam, the radial distribution of electrons in the beam, and the angular divergence of this

138 beam. Clearly, in order to generate a clinically realistic MC simulation of dose delivery from

139 any individual accelerator, the parameters of the model of the electron beam must be

140 determined specifically for that accelerator [11].

141 For above-discussed reasons, availability of a well-defined and realistically

142 executable procedure of specifying the MC model of the primary electron beam in an

143 individual linear accelerator is of major importance in the subsequent application of MC-

144 based modelling of clinical procedures using this particular accelerator. Notably, parameters

145 of a MC model of the electron beam of a linac can only be determined indirectly by analysing

146 a number of depth and lateral dose-profiles measured in specified conditions, best in a

147 standard water phantom.

148 Due to the fundamental importance of this issue, several studies have been published

149 proposing various experimental setups and methods of such analysis [6-9,11-18]. In the study

150 of Almberg et al. [9] an algorithm is proposed to determine values of two parameters (nominal 
151 energy and spot size) of the primary electron beam model, basing on depth and lateral photon

152 beam profiles measured for three fields: $2 \times 2,10 \times 10$, and $20 \times 20 \mathrm{~cm} 2$. The MC profiles are

153 calculated for preselected values of nominal energies and spot sizes. The authors propose an

154 ad hoc cost function, calculated from a range of hand-crafted features extracted from profiles,

155 to select between some preselected nominal energy and spot size values those which minimize

156 the cost function. The algorithm, iterating over preselected values, finds values of only two of

157 the four model parameters. The tuning procedure proposed in the study of Pena et al. [12] is

158 aimed at determining three parameters (the nominal energy, spot size, and angular divergence)

159 of the primary electron beam model, basing on a depth and a penumbra profile for the $5 \times 5$

$160 \mathrm{~cm} 2$ field and three lateral profiles for four fields: 5x5, 10x10, 20x20, and 40x40 cm2. The

161 MC profiles are also calculated for preselected values of nominal energies and an arbitrarily

162 fixed values of spot size and angular divergence. In the first step, the authors, using these

163 fixed angular divergence and spot size values and applying a figure of merit, search for an

164 optimal energy, iterating over MC results. Only the first step of this tuning procedure is

165 formalized, because in the next step, after fixing the energy parameter to the value found in

166 the previous step, an optimal spot size is searched for by trial and error, comparing measured

167 and simulated penumbra for a $5 \times 5 \mathrm{~cm} 2$ field. In the last step, the value of the angular

168 divergence parameter is searched for, also by trial and error, using lateral profiles. The last

169 two steps require an extensive MC simulation effort while being based on a trial-and-error

170 approach. Other studies of [13-18] are also based on a trial-and-error approach which, while

171 being computationally demanding, offers no systematic procedure for estimating parameter

172 values in the applied model of the primary electron beam.

173 It follows that in the published papers describing tuning the model of the primary

174 electron beam, ad hoc approaches are primarily used. In most cases a trial-and-error approach

175 has been adopted. Other approaches rely on of searching for the beam model parameters using 
176 various figures of merit, over a predefined set of parameters for which MC results have been

177 pre-computed. Within some hybrid approaches, the value of one of the model parameters is

178 found by searching over a set of preselected values while values of the remaining parameters

179 are found by trial and error - which again requires extensive MC simulation.

180 The published studies generally share common feature - they lack any

181 phenomenological model enabling the values of primary electron beam parameters to be

182 estimated directly from the measured dose profiles. They also suffer from specific, often quite 183 demanding requirements with respect to experimental equipment and measurement 184 procedures. As different measurement devices and different dosimetry procedures are 185 typically used at various clinical centres, relaxing such constraints is highly desirable.

186 In contrast to such studies, we propose a flexible framework, termed DeepBeam, such

187 that its user may collect the profile data using dosimetry tools and protocols at her/his disposal 188 and select the profiles to be measured according to her/his best experience. Then, a regression 189 model is created which can be directly and routinely used to estimate the parameters of the 190 model of the primary electron beam. The proposed framework, apart from tuning electron 191 beams of Monte Carlo simulators of real linear accelerators and, possibly, consecutive in 192 silica designs of radiotherapy procedures, may also be applied in routine quality assurance of 193 an operating linear accelerator - not only to verify its beam stability via dose profile analysis, 194 but also to indicate which of the beam parameters had likely changed and by how much. The 195 complete code of this framework and the data used for training the regression models are 196 freely available at https://github.com/taborzbislaw/DeepBeam .

198 II. Material and methods

199 II.1. The MC simulator and sources of data 
200 While the proposed framework for tuning primary electron beams of MC simulators of linear

201 accelerators can be used for any such simulator, the present study is based on data generated

202 by the PRIMO simulator, version 0.1.5.1307 [19] (www.primoproject.net). PRIMO is a

203 freely-distributed application used for simulating dose delivery during radiotherapy $[8,18,19]$.

204 It is based on the PENELOPE 2011 [20] general purpose Monte Carlo engine and allows

205 simulation of dose delivery to be performed for some other linear accelerator models, based

206 on their geometry, as provided by their manufacturers. This last feature is especially

207 important, as details of accelerator geometry are usually confidential and may not be available

208 from the manufacturer, even upon request. Hence, using PRIMO, attention could be focussed

209 on the primary goal of designing a framework for tuning the electron beam of this simulator

210 without undue concern with simulation details related to the physics, materials or the

211 geometry configuration of the simulated accelerator system.

212 All simulations were run using the PRIMO Varian Clinac 2300 C/D simulator

213 operating in photon mode at a nominal energy of $6 \mathrm{MV}$. Electron beam simulation in PRIMO

214 is configured by specifying values of four beam parameters: $E$ - the initial electron beam

215 energy (in $\mathrm{MeV}$ ), $\sigma_{E}$ - the full-width-at-half-maximum (FWHM) of the primary beam energy

216 distribution (in $\mathrm{MeV}$ ), $s$ - the focal spot FWHM (in $\mathrm{cm}$ ), and $\alpha$ - the angular beam divergence

217 (in degrees). The developed framework should however be readily adaptable if different

218 primary beam parameters were specified in the PRIMO simulator, or if other MC simulators

219 of linear accelerators were applied.

\section{II.1.1. Simulated input data}

222 To generate training data for the machine learning framework, the simulations were run for a 223 total of 300 tuples $\left(E, \sigma_{E}, \mathrm{~s}, \alpha\right)$ within the set $S$ such that: 


$$
\begin{gathered}
S=\left\{\left(E, \sigma_{E}, S, \alpha\right): E \in\{5.6,5.8,6.0,6.2,6.4\}, \sigma_{E} \in\{0.0,0.5,1.0\}\right. \\
S \in\{0.0,0.1,0.2,0.3,0.4\}, \alpha \in\{0,1,2,3\}\}
\end{gathered} .
$$

227 At the first simulation stage, $10^{8}$ histories (a history corresponds to a single electron of the

228 virtual primary beam) were simulated for each tuple $\left(E, \sigma_{E}, \mathrm{~s}, \alpha\right)$ and the phase-space file 229 (PSF) above the secondary collimators was saved for further purposes. At this first stage, the

230 splitting roulette variance reduction technique [21] was used with the size of the splitting

231 region set to the largest region, i.e. to the $40 \times 40 \mathrm{~cm}^{2}$ field. The saved PSFs were then used to

232 simulate dose delivery to a homogeneous cubic water phantom for three fields: $3 \times 3 \mathrm{~cm}^{2}$,

$23310 \times 10 \mathrm{~cm}^{2}$, and $30 \times 30 \mathrm{~cm}^{2}$. The size of the phantom was set to $50 \times 50 \times 50 \mathrm{~cm}^{3}$. The doses in

234 the phantom were tallied within a regular grid of $0.5 \times 0.5 \times 0.5 \mathrm{~cm}^{3}$ voxels. The respective

235 faces of the phantom were set parallel to the respective main axes of the coordinate frame of

236 reference of the accelerator. The main axis of the phantom coincided with the photon beam

237 axis. The source-to-surface distance $(\mathrm{SSD})$ was set at $100 \mathrm{~cm}$, the isocentre being located at

238 the front surface of the phantom. Splitting in the water phantom was selected as the variance

239 reduction method [21] at this simulation stage, with a splitting factor of 300 . The uncertainty

240 of the dose values tallied in the water phantom always remained within $1.5 \%$. The calculated

$2413 \mathrm{D}$ spatial distribution of doses within the phantom was saved to a text file, separately for

242 each tuple $\left(E, \sigma_{E}, \mathrm{~s}, \alpha\right)$ and for each field. A total of $9003 \mathrm{D}$ dose files were collected. Each

243 3D dose file contained $10^{6}$ dose values measured at $(x, y, z)$ coordinates given by the following

244 coordinate ranges:

$x \in\{-25+0.25+0.5 * i, i=1 \ldots 100\}$

$246 y \in\{-25+0.25+0.5 * j, j=1 \ldots 100\}$,

$$
z \in\{0.25+0.5 * k, k=1 \ldots 100\}
$$


248 where the $z$ axis is parallel to the radiation field axis. To generate testing data for the machine

249 learning framework, the simulations were run further for 25 tuples $\left(E, \sigma_{E}, \mathrm{~s}, \alpha\right)$ with primary

250 beam parameters sampled as follows:

251

252

$$
\begin{gathered}
E \in\{5.65+i \cdot 0.05, i=0 \ldots 14\} \backslash\{5.8,6.0,6.2\} \\
\sigma_{E} \in\{0.1+i \cdot 0.1, i=0 \ldots 8\} \backslash\{0.5\} \\
s \in\{0.05+i * 0.1, i=0 \ldots 3\} \\
\alpha \in\{0.5+i * 0.25, i=0 \ldots 9\} \backslash\{1,2\}
\end{gathered}
$$

254 Applying the above sampling scheme, it was assured that the primary electron beam

parameters $\left(E, \sigma_{E}, \mathrm{~s}, \alpha\right)$ in the testing set never coincided with parameters used for generating

256 the training set, and consequently, that the electron beam parameters for the testing set were

257 well separated from the electron beam parameter selected for training.

All simulations were run using the PlGrid infrastructure (Prometheus grid,

https://kdm.cyfronet.pl/portal/Main_page) and required a total real time of about 2.5 months.

260 During the simulation period 12 Prometheus nodes run the PRIMO software, each node 261 equipped with two Intel Xeon E5-2680v3 processors, 24 cores in total, and 128 GB RAM.

262 The simulation of a single case, i.e., of three fields for a single tuple $\left(E, \sigma_{E}, \mathrm{~s}, \alpha\right)$, required

263 about 40 CPU hours. As the operating system installed on the nodes is Linux CentOS 7, while

264 PRIMO is a Windows application, wine software (https://www.winehq.org/) was installed and 265 configured in order to use PRIMO in graphic mode under Linux exactly as if Windows were 266 the operating system.

\section{II.1.2. Measured input data}

269 Dose profiles were measured in water for the $6 \mathrm{MV}$ photon beam of a clinically exploited 270 Clinac 2300C/D medical accelerator at the Krakow Branch of the National Research Institute 271 of Oncology. A PTW MP3 Water Phantom and PTW Markus Type 23343 and PTW Semiflex 
272 Type 31010 ionization chambers were used for dosimetry. PTW Mephysto software was

273 applied for data collection. Three experimental setups of dose profile measurements were

274 arranged, as described in more detail in the Results section.

\section{II.2. Applied models and computational framework}

277 The task to solve is a regression problem, i.e., given dose profiles in a water phantom, the 278 parameters $\left(E, \sigma_{E}, \mathrm{~s}, \alpha\right)$ of the primary electron beam are to be estimated. To prepare training 279 and test data, each 3D dose spatial distribution was normalized to the dose value calculated 280 along the photon beam axis at the depth of maximum $\left(D_{\max }=1.4 \mathrm{~cm}\right)$, which was then set to $281100 \%$ (such normalization is not essential if not implemented in a clinical measurement 282 system). Next, from each 3D dose file six profiles were extracted: one depth profile along the 283 axis of the radiation field, and five lateral profiles at depths: $1.4 \mathrm{~cm}, 5 \mathrm{~cm}, 10 \mathrm{~cm}, 20 \mathrm{~cm}$, and

$28430 \mathrm{~cm}$. To match the resolution of the simulated profiles and the typical spatial resolution of 285 clinical dosimetry systems (usually $1 \mathrm{~mm}$ ), linear interpolation was applied to the tallied 286 simulated doses during profile extraction. Additionally, as PRIMO assumes the electron beam 287 spot to be of circular shape, the lateral dose profiles extracted from the 3D dose files consisted 288 of averages over two perpendicular lateral dose profiles over the $x$ and $y$ directions. Such 289 averaging is not a necessary condition and may be skipped if a more complex, e.g., elliptic, 290 electron spot shape is assumed by the accelerator simulator.

291 The extracted dose profiles (18 profiles for each tuple $\left(E, \sigma_{E}, \mathrm{~s}, \alpha\right)$ ) represent a 292 reasonable maximum set $\operatorname{Prof}_{M A X}$ of dose profiles to be used in the proposed machine learning 293 framework. Moreover, the extracted depth dose profiles span the range of $\mathrm{z} \in<0.3 \mathrm{~cm}, 49.7$ $294 \mathrm{~cm}>$, while all the extracted lateral dose profiles span the range of $\mathrm{x} \in<-24.7 \mathrm{~cm}, 24.7 \mathrm{~cm}\rangle$, 295 i.e., the maximum ranges for the geometry of the simulated water phantom and for the spatial 296 resolution of the grid of tallied dose values. 
The proposed framework is customizable, meaning that any subset of the dose profiles

298 can be selected from the complete set of dose profiles to match the needs of an individual

299 user. The ranges over which the profiles are measured can also be arbitrarily selected to match

300 the measurement ranges of real profiles. For example, the user may decide to build her/his

301 regression model which predicts the parameters of the model of the electron beam $\left(E, \sigma_{E}, \mathrm{~s}\right.$,

$302 \alpha$ ) from the depth dose profiles and from lateral dose profiles at $10 \mathrm{~cm}$ depth, all collected for

$30310 \times 10 \mathrm{~cm}^{2}$ and $30 \times 30 \mathrm{~cm}^{2}$ fields, depth dose profiles measured up to $35 \mathrm{~cm}$, and lateral dose

304 profiles measured over the ranges between $-10 \mathrm{~cm}$ to $+10 \mathrm{~cm}$ and between $-20 \mathrm{~cm}$ to $+20 \mathrm{~cm}$,

305 for $10 \times 10 \mathrm{~cm}^{2}$ and $30 \times 30 \mathrm{~cm}^{2}$ fields, respectively. Given these user-defined constraints the

306 framework finds the optimum regression model, as described in the following sections.

308 II.2.1. PCA + SVR regression model

309 Let Prof $=\left\{\operatorname{Prof}_{1}, \operatorname{Prof}_{2}, \ldots, \operatorname{Prof}_{\mathrm{n}}\right\}$ represent a user-selected subset of Prof $f_{\mathrm{MAX}}$. A user-

310 selected spatial range Range $e_{i}$ is associated with each $\operatorname{Prof}_{i}$ (Range $e_{i}$ would typically be the 311 user-dependent spatial range over which $\operatorname{Prof}_{i}$ is measured under clinical settings). Each

312 subscript $i$ corresponds to a unique field size and a unique dose profile type (either depth or

313 lateral, at one of the five depths: $D_{\max }=1.4 \mathrm{~cm}, 5 \mathrm{~cm}, 10 \mathrm{~cm}, 20 \mathrm{~cm}$, or $30 \mathrm{~cm}$ ).

314 As each dose profile $\operatorname{Prof}_{i}$ is sampled within a given spatial resolution (usually $1 \mathrm{~mm}$ ),

315 it may be considered a 1D vector of some dimensionality (dependent of the sampling 316 resolution and the sampling range Range $_{i}$ ). The regression task which is to be solved can be 317 formulated as follows:

$319 \operatorname{Par}=f_{\text {Par }}\left(\operatorname{Prof}_{1}, \operatorname{Prof}_{2, \ldots}, \operatorname{Prof}_{n}\right)+\epsilon_{\text {Par }}$, 
321 where Par is any element of the tuple $\left(E, \sigma_{E}, \mathrm{~s}, \alpha\right), f_{P a r}$ is the regression function and $\varepsilon_{P a r}$ is

322 the residual term. The components of each $\operatorname{Prof}_{i}$ are however strongly correlated as they

323 represent dose values measured at neighbouring spatial locations. For this reason, the model

324 given by Eq. (4) may not be very effective, as the set of explanatory variables (arguments of

$\left.325 f_{\text {Par }}\right)$ contains a high contribution of redundant information.

To resolve this redundancy problem dimensionality reduction is applied. Typically,

dose profiles are specified by applying some ad-hoc features, such as width at half maximum,

328 width of penumbra regions, "wing heights" in lateral profiles, etc. Here, rather than rely on

329 such hand-crafted features, Principal Component Analysis (PCA) is applied to the analysed

330 profiles [22]. PCA will then find uncorrelated features which form a linear combination of the

331 original features (dose values). Moreover, each PCA feature is assigned a percentage of the

332 total variance of profile shapes it explains. As demonstrated in what follows, three most

333 important PCA features usually explain over $98 \%$ of the variability of shapes of the training

334 dose profiles. PCA reduces the dimensionality of the space of explanatory variables by a

335 factor of $10^{2}$ - the final set of features consists of $3 n$ elements (explanatory variables) - three

336 features for each profile Prof $_{i}$ in Prof. The learnt PCA models were saved in respective files (a

337 separate PCA model $M_{P C A, i}$ file for each index $i$ ) and subsequently used at the stage of model 338 testing.

Clearly, for each $i$ - index there are 300 training profiles $\left\{\operatorname{Prof}_{i, 1}, \operatorname{Prof}_{i, 2}, \ldots, \operatorname{Prof}_{i, 300}\right\}$

340 corresponding to 300 different tuples $\left\{\left(E, \sigma_{E}, \mathrm{~s}, \alpha\right)_{1},\left(E, \sigma_{E}, \mathrm{~s}, \alpha\right)_{2}, \ldots,\left(E, \sigma_{E}, \mathrm{~s}, \alpha\right)_{300}\right\}$ and a

341 single PCA model $M_{P C A, i}$ which extracts three features $\left(F_{i, 1}, F_{i, 2}, F_{i, 3}\right)_{k}$ from Prof $_{i, k}$. Hence, the 342 regression problem, after dimensionality reduction, becomes:

$344 \operatorname{Par}=f_{P A R}^{P C A}\left(F_{1,1}, F_{1,2}, F_{1,3}, F_{2,1}, F_{2,2}, F_{2,3}, \ldots, F_{n, 1}, F_{n, 2}, F_{n, 3}\right)+\epsilon_{P C A}$, 
346 where Par is any element of the tuple $\left(E, \sigma_{E}, \mathrm{~s}, \alpha\right), f_{P A R}^{P C A}$ is the PCA-based regression function

347 and $\varepsilon_{P C A}$ is the residual term. To learn the regression functions, the following training set, $\operatorname{Tr}$,

348 was applied:

$350 \operatorname{Tr}=\left\{\left(E, \sigma_{E}, S, \alpha\right)_{K},\left\{\left(F_{1,1}, F_{1,2}, F_{1,3}\right)_{K^{\prime}}\left(F_{2,1}, F_{2,2}, F_{2,3}\right)_{K^{\prime}} \ldots,\left(F_{n, 1}, F_{n, 2}, F_{n, 3}\right)_{K}\right\}, K=\right.$

$351 \quad 1 . .300\} .(6)$

353 Support Vector Regression (SVR) with rbf kernel was selected as the regressor [23] though

354 other options are also available. The best regression models were selected using a 5-fold cross 355 validation run on $\operatorname{Tr}$. After training, four $f_{P A R}^{P C A}$ regressors were obtained, one per $E, \sigma_{E}$, s, and $356 \alpha$. The regression models were saved to files and subsequently used in testing.

\section{II.2.2. The Deep Learning regression model}

359 The processing pipeline described in the previous section consists of two separate steps: 360 feature extraction, and training of four regressors. In the current section an end-to-end regression model is described which, during training, learns both dose profile data representation and regression functions simultaneously for all primary beam parameters $(E$, $\left.\sigma_{E}, \mathrm{~s}, \alpha\right)$. The model presented here is based on deep learning. The architecture of the deep learning (DL) model is outlined in Fig. 1. described in the previous section. In short, each $\operatorname{Prof}_{i}$ in Prof is a separate input for the DL

367 model and is processed by a separate encoder block. Each encoder block consists of a few 368 convolution blocks. Each convolution block consists of two 1D convolutions (filter size equal 369 to 3 , number of filters equal to 16,32 , 64 , etc. in the consecutive convolution blocks, ReLU 
activation) followed by a MaxPool1D layer which reduces the size of the data by a factor of two. The number $L$ of convolution blocks in each encoder block is selected based on the

372 length $N$ of the input of this block, according to the formula $L=\operatorname{int}\left(\log _{2} N / 3\right)$, i.e., the number

373 of features learnt by any encoder block cannot be less than 3. Each encoder block ends with

374 1D convolution with a single filter of unit size. The outputs of the encoder blocks are then

375 concatenated to form a 1D vector of features (in analogy to PCA features). This feature vector

376 is then processed by two fully connected layers of size 100 and ReLU activation. The output

377 of the last fully connected layer is next fed into the final fully connected layer with four

378 outputs and no activation. These outputs are expected to deliver estimates of $E, \sigma_{E}, \mathrm{~s}$, and $\alpha$.

379 The training data $\operatorname{Tr}_{D L}$ for the DL model is:

$\operatorname{Tr}_{D L}=\left\{\left(E, \sigma_{E}, s, \alpha\right)_{K},\left\{\operatorname{Prof}_{1, K}, \operatorname{Prof}_{2, K}, \ldots, \operatorname{Prof}_{n, K}\right\}, K=1 \ldots 300\right\}$

383 The model is trained for 300 epochs using the Adam optimizer and a constant learning rate equal to 0.0001 . The loss function selected for this regression problem was mean square error between the model outputs and ground truth data. A $20 \%$ portion of the training set was randomly selected for model validation. The best model found during training was saved to a

387 file and used in subsequent testing.

\section{II.2.3. Testing the models}

390 At the stage of model testing, the testing profiles were fed at the input of either the PCA+SVR 391 or DL models. The PCA+SVR model first extracts the features from the testing profiles based 392 on PCA models learnt on the training set. These test features are then processed by SVR 393 regressors which return the predicted values of $E, \sigma_{E}, \mathrm{~s}$, and $\alpha$. In the case of the DL model 394 the raw testing profiles are fed at the input of the DL model which returns the predicted values 
of $E, \sigma_{E}, \mathrm{~s}$, and $\alpha$. The true and predicted values of $E, \sigma_{E}, \mathrm{~s}$, and $\alpha$ are then compared using correlation analysis and linear regression.

\section{II.2.4. Optimizing the solution with profiles reconstructed from regression results}

398 The regression results can be further improved by minimizing the difference between the

399 actual profiles being fed at the input of regressors and profiles reconstructed from the 400 regression results. In particular, the parameters of the model of the primary electron beam for 401 the training set $\operatorname{Tr}$ (Eq. (6)) have the form of a regular grid $S$, defined in Eq. (1), embedded 402 within a 4D hypercube $H$. With every node $Q$ of $S$ associated are PCA features corresponding 403 to the dose profiles determined for primary electron beam model parameters $\left(E, \sigma_{E}, \mathrm{~s}, \alpha\right)_{\mathrm{Q}}$ 404 assigned to $Q$. The regressions return a point $P=\left(E, \sigma_{E}, \mathrm{~s}, \alpha\right)_{\mathrm{PRED}}$ within $H$ (see Fig. 2 for a 405 2D example). Consecutively, using interpolation, PCA features corresponding to $P$ may be 406 determined, and next an inverse PCA transform applied to them in order to reconstruct 407 profiles from the results of regression models $\left(E, \sigma_{E}, \mathrm{~s}, \alpha\right)_{\text {PRED. Thus, for each } \text { Prof }_{i} \text { in Prof a }}$ 408 reconstructed profile $\operatorname{RecProf}_{i}(P)$ obtains, which in general differs from $\operatorname{Prof}_{i}$. This difference 409 can then be further minimized using one of several optimization methods, with $\left(E, \sigma_{E}, \mathrm{~s}\right.$, $410 \alpha)_{\mathrm{PRED}}$ as the starting point for such minimization. Namely, beginning with $P=\left(E, \sigma_{E}, \mathrm{~s}\right.$, $411 \alpha)_{\mathrm{PRED}}, P_{M I N}=\left(E, \sigma_{E}, \mathrm{~s}, \alpha\right)_{\mathrm{MIN}}$ is sought, such that:

$$
P_{M I N}=\underset{P \in H}{\operatorname{argmin}} \sum_{i=1}^{n} w_{i}\left\|\operatorname{Prof}_{i}-\operatorname{RecProf}_{i}(P)\right\|^{2},
$$

415 where $w_{i}$ is the weight assigned to the $i$-th profile. In the experiments all $w_{i}$ were set to unity 416 but in general the user may set these according to her/his actual needs. The minimization 417 problem defined in Eq. (8) was solved using the SLSQP method [24]. 
420 All models were implemented in Python 3.6.10. The scipy library (version 1.5.2) was used to

421 implement regression models using PCA and SVR. The same library was used to run 422 interpolation over 3D dose distributions to extract dose profiles, optimization of the regression

423 results and profile reconstruction from regression results. The DL model was implemented 424 using the keras (version 2.3.1) library. All codes, pretrained models, as well as training and 425 testing data, are freely available at https://github.com/taborzbislaw/DeepBeam .

427 III Results

\section{III.1. Analysis of simulated training data}

429 Detailed analysis of simulated training data formed the basis for model design decisions and 430 aided in selecting the best hyperparameters for the developed model. Following these 431 decisions, a final verification of the performance of the model was performed using only test 432 data. Thus, in accordance with the fair model selection principle, test data were never used in 433 model fine-tuning.

434 The first issue considered was what number of PCA features extracted from the 435 profiles explains what fraction of the variability in the shapes of profiles. Results are shown in 436 Fig. 3a separately for three analysed fields $\left(3 \times 3 \mathrm{~cm}^{2}, 10 \times 10 \mathrm{~cm}^{2}\right.$ and $\left.30 \times 30 \mathrm{~cm}^{2}\right)$ as averages over six profiles extracted from the simulated 3D dose distributions for each of these three 438 fields (depth profile and five lateral profiles at depths $D_{\max }=1.4 \mathrm{~cm}, 5 \mathrm{~cm}, 10 \mathrm{~cm}, 20 \mathrm{~cm}$, and $43930 \mathrm{~cm}$ ). The error bars represent standard deviations of the values of explained variance, 440 calculated over the six profiles of these three fields. Clearly, three PCA features suffice in 441 explaining most of the variability of the shapes of profiles, so any further increase of PCA 442 features above that number would not offer much in terms of added benefit.

443 Results shown in Fig. 3b illustrate the relative contributions that each of the three 444 most important PCA features introduce in explaining the variance of profile shape. It is 
445 evident that the first PCA feature explains most of the variation in the depth profile (ID=1).

446 The contributions offered by consecutive PCA features in explaining the overall variation in 447 the shapes of lateral profiles increase with increasing depth at which these lateral profiles are 448 extracted. It follows from this figure that use of lateral profiles extracted at larger depths 449 offers more benefit in model predictions, as the variability of their shapes is richer (i.e., the 450 contribution of higher PCA modes to the shape of the final profile is then more pronounced). 451 Effectively, more information about the primary electron beam may be encoded in such 452 profiles at larger depths.

The impact of the three major PCA features on the shape of dose profiles is shown in

454 Fig. 4 for two selected profiles $\left(10 \times 10 \mathrm{~cm}^{2}\right.$ lateral profile at $30 \mathrm{~cm}$ depth - upper panels, and $45510 \times 10 \mathrm{~cm}^{2}$ depth profile - lower panels). Here, changes with respect to the mean profile 456 shapes when varying any one of the three most important PCA features are shown. The PCA 457 features of mean profiles are all equal to zero. Profiles marked in this figure as "Negative 458 feature" have only the first, second, or third PCA feature negative in plots within the left, 459 middle, or right panels, respectively. Similarly, profiles marked as "Positive feature" have 460 only the first, second, or third PCA feature positive in respective left, middle, or right panels. The coefficient of determination between ground truth and predicted values of energy, 462 spot size, and angular divergence is shown in Fig. 5. The coefficient of determination was 463 calculated for held-out parts of the full training set, in accordance with cross-valdation 464 approach. Results presented in this figure are obtained for regressors trained on different 465 numbers of profiles of any of the three fields: $3 \times 3 \mathrm{~cm}^{2}, 10 \times 10 \mathrm{~cm}^{2}$, and $30 \times 30 \mathrm{~cm}^{2}$. For 466 example, in the case of the number of profiles of each field being equal to one, only depth 467 profiles of the three fields were used for regressor training. For the number of profiles of each 468 field equal to two, depth profiles and lateral profiles at $1.4 \mathrm{~cm}$ depth of these three fields were 469 used for regressor training. It follows from the results shown in this figure that a total of six 
470 profiles (one depth and one lateral), that is two from each of three $\left(3 \times 3 \mathrm{~cm}^{2}, 10 \times 10 \mathrm{~cm}^{2}\right.$ and

$47130 \times 30 \mathrm{~cm}^{2}$ ) fields, would suffice in precisely predicting the values of $E$, s, and $\alpha$.

472 Note that in Fig. 5 predictions of the FWHM of the primary beam energy distribution,

$473 \sigma_{E}$, have not been presented, as it was not possible to train a regressor to precisely predict the

474 value of $\sigma_{E}$. The effect of varying the value of $\sigma_{E}$ on the shapes of the profiles was noted to be

475 very small - below $2 \%$, in line with the noise level, which is the likely cause of lack of

476 success in training regressors to predict the value of the parameter which represents the 477 energy distribution of the primary beam. Thus, only $E$, s, and $\alpha$ (energy, spot size, and angular 478 divergence) were further considered in the analysis.

479 The coefficient of determination between ground truth and predicted values of energy,

480 spot size, and angular divergence for a limited selection of profiles (depth, lateral at depth of $481 \mathrm{D}_{\max }=1.4 \mathrm{~cm}$ and lateral at depth of $10 \mathrm{~cm}$ ) and a varied number of fields for these profiles 482 was also analysed. Application of a total of six profiles - one depth profile and two lateral 483 profile, and any two of three fields $\left(3 \times 3 \mathrm{~cm}^{2}, 10 \times 10 \mathrm{~cm}^{2}\right.$, or $\left.30 \times 30 \mathrm{~cm}^{2}\right)$ would be sufficient in 484 obtaining precise predictions of $E, \mathrm{~s}$, and $\alpha$ values. It appears however that three profiles 485 extracted from a single field (except for the $3 \times 3 \mathrm{~cm}^{2}$ field alone), would also be sufficient to 486 precisely determine the values of primary electron beam parameters $E$, s, and $\alpha$, with a 487 coefficient of determination above 0.98 .

488 Considering the above-discussed results obtained using training, the final design 489 decision was made to train regressors based on PCA features extracted from a total of six 490 profiles, i.e., three profiles (depth, lateral at $\mathrm{D}_{\max }=1.4 \mathrm{~cm}$ depth and lateral at $10 \mathrm{~cm}$ depth) of 491 two field sizes $\left(10 \times 10 \mathrm{~cm}^{2}\right.$ and $\left.30 \times 30 \mathrm{~cm}^{2}\right)$.

492 The testing results for the PCA+SVR model are shown in Fig. 6. Models trained on 493 the training data were applied to previously unseen testing data and the predicted values of 494 primary beam parameters compared to the ground truth data, i.e., the values of primary beam 
495 parameters applied in the generation of simulated profiles. Notably, the values of the 496 coefficient of determination for the testing data were only slightly lower than those obtained

497 for the training data - implying that the regressors were not overfitted. Also shown in this

498 figure are best-fitted linear regression lines to demonstrate the precision with which the model 499 is able to predict the primary beam parameters. The slopes of these regression lines are all 500 close to 1.0. The prediction errors were estimated as values of standard deviation of the 501 difference between the true and predicted values of the primary electron beam parameter, and 502 were equal to $0.03 \mathrm{MeV}, 0.007 \mathrm{~cm}$, and $0.13^{\mathrm{O}}$ for $E$, s, and $\alpha$, respectively.

503 The testing results for the deep learning model trained on the same set of profiles as

504 those used for the PCA+SVR model are shown in Fig. 7. The results for the deep learning 505 model are slightly inferior to those obtained for the PCA+SVR model which is not surprising, 506 since the deep model was trained only on 300 sets of profiles, which may not be sufficient for 507 a deep learning task. Further refinements would certainly be possible, however in that case 508 more data would need to be generated. Yet, as demonstrated in the next section, both models 509 offer good starting points for optimization-based estimates of the parameters of a model of the 510 primary electron beam from clinical measurements, leading to virtually the same final results.

511 The prediction errors for the deep model were equal to $0.065 \mathrm{MeV}, 0.023 \mathrm{~cm}$, and $0.21^{\mathrm{O}}$ for $E$,

512 s, and $\alpha$, respectively.

513 Discrepancies between the slopes of the best fit lines and the ideal 1.0 value are due to 514 the noise present in the training data, which, although being relatively low $(1.5 \%)$ is however 515 higher than that in real measurements. Decreasing the noise level to $0.5 \%$ would however 516 increase the computation time by a factor of 10 which is unrealistic in view of the 517 computational expense. The optimization procedure which follows the regression, as 518 described in the previous section, resolves this issue.

519 III.2. Analysis of clinical data 
520 The developed framework was used to find the values of primary electron beam parameters

521 which could best reproduce real profiles measured using the $6 \mathrm{MV}$ photon beam of a Clinac

522 2300C/D medical accelerator in a PTW MP3 Water Phantom. The applied input fields and

523 profiles, obtained beam parameters, and mean errors of the reconstructed dose distributions

524 against those measured, for three cases of experimental setups discussed below, are gathered

525 in Table 1. The measured and reconstructed profiles for the respective sets of input profiles in

526 each of these three cases are compared in Fig. 8.

528 Table 1. Results of SVR regression and deep model analysis of clinical profile data.

\begin{tabular}{|c|c|c|c|c|c|c|}
\hline \multirow{2}{*}{$\begin{array}{c}\text { Case } \\
\text { ID }\end{array}$} & \multirow{2}{*}{$\begin{array}{c}\text { Applied } \\
\text { Fields }\end{array}$} & \multirow[b]{2}{*}{ Applied Profiles } & \multicolumn{3}{|c|}{ Beam parameters } & \multirow{2}{*}{$\begin{array}{c}\text { Mean absolute error between } \\
\text { measured and reconstructed } \\
\text { profiles }[\%]\end{array}$} \\
\hline & & & $\begin{array}{c}\text { SVR regression } \\
\text { E }_{\text {PRED }} \\
\text { SPRED, } \\
\text { a }_{\text {PRED }}\end{array}$ & $\begin{array}{c}\text { DL regression } \\
\text { E }_{\text {PRED }} \\
\text { SPRED } \\
\text { a }_{\text {PRED }}\end{array}$ & $\begin{array}{c}\text { Final estimation } \\
\text { E }_{\text {FINAL }}, \\
\text { S FINAL }, \\
\mathbf{a}_{\text {FINAL }}\end{array}$ & \\
\hline \multirow{5}{*}{1} & & Depth profile & \multirow{5}{*}{$\begin{array}{l}5.54 \mathrm{MeV}, \\
0.0 \mathrm{~cm}, \\
1.97^{\mathrm{O}}\end{array}$} & \multirow{5}{*}{$\begin{array}{l}6.02 \mathrm{MeV}, \\
0.0 \mathrm{~cm}, \\
2.35^{\circ}\end{array}$} & \multirow{5}{*}{$\begin{array}{l}5.86 \mathrm{MeV}, \\
0.0 \mathrm{~cm}, \\
2.44^{\mathrm{O}}\end{array}$} & 0.33 \\
\hline & & Lateral at Dmax & & & & 0.94 \\
\hline & \multirow{2}{*}{$10 \times 10 \mathrm{~cm}^{2}$} & Depth profile & & & & 0.50 \\
\hline & & Lateral at Dmax & & & & 1.03 \\
\hline & $30 \times 30 \mathrm{~cm}^{2}$ & Lateral at Dmax & & & & 0.90 \\
\hline \multirow{4}{*}{2} & \multirow{2}{*}{$10 \times 10 \mathrm{~cm}^{2}$} & Depth profile & \multirow{4}{*}{$\begin{array}{l}5.47 \mathrm{MeV}, \\
0.23 \mathrm{~cm} \\
2.08^{\circ}\end{array}$} & \multirow{4}{*}{$\begin{array}{l}5.50 \mathrm{MeV}, \\
0.18 \mathrm{~cm} \\
2.41^{\mathrm{O}}\end{array}$} & \multirow{4}{*}{$\begin{array}{l}5.60 \mathrm{MeV}, \\
0.25 \mathrm{~cm} \\
2.39^{\circ}\end{array}$} & 0.33 \\
\hline & & Lateral at $10 \mathrm{~cm}$ depth & & & & 0.41 \\
\hline & \multirow{2}{*}{$30 \times 30 \mathrm{~cm}^{2}$} & Lateral at Dmax & & & & 0.49 \\
\hline & & Lateral at $10 \mathrm{~cm}$ depth & & & & 0.26 \\
\hline \multirow{3}{*}{3} & \multirow{3}{*}{$10 \times 10 \mathrm{~cm}^{2}$} & Depth profile & \multirow{3}{*}{$\begin{array}{l}5.43 \mathrm{MeV} \\
0.24 \mathrm{~cm} \\
1.95^{\circ}\end{array}$} & \multirow{3}{*}{$\begin{array}{l}5.62 \mathrm{MeV}, \\
0.27 \mathrm{~cm} \\
2.47^{\mathrm{O}}\end{array}$} & \multirow{3}{*}{$\begin{array}{l}5.60 \mathrm{MeV}, \\
0.24 \mathrm{~cm} \\
2.41^{\mathrm{O}}\end{array}$} & 0.35 \\
\hline & & Lateral at Dmax & & & & 0.75 \\
\hline & & Lateral at $10 \mathrm{~cm}$ depth & & & & 0.40 \\
\hline
\end{tabular}




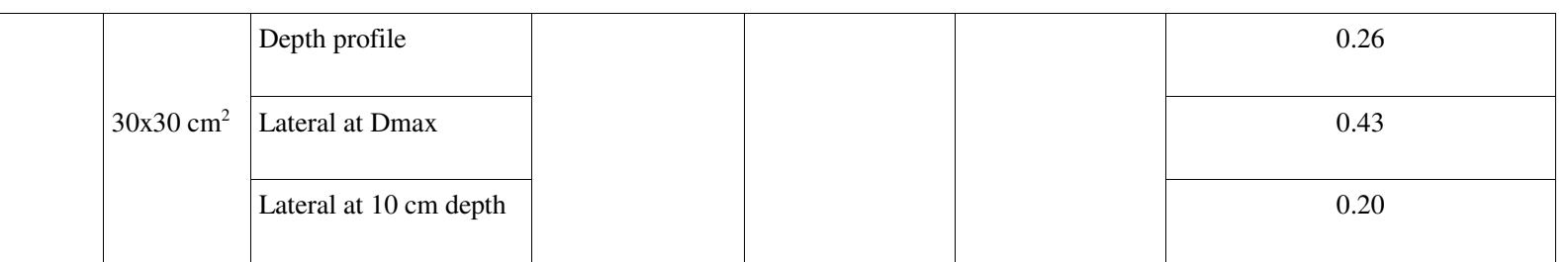

529 Table legend: The results for three experimental setups (Cases 1-3)) are reported in the table.

530 The applied input fields and profiles are shown in columns 2 and 3. Values of PCA+SVR or

531 DL- predicted beam parameters are shown in in columns 4 and 5. The mean absolute

532 differences between the measured profiles and profiles reconstructed using final estimation

533 parameters are shown in the last column.

Three experimental design cases were investigated, where different sets of measured profiles (as shown in the second and third columns of Table 1) were used as input. In each of

537 the three cases, the models were trained on a set of training profiles corresponding to those 538 measured, after suitable adjustment of the ranges of the training profiles. Following this 539 training, the measured profiles were then input to the trained PCA+SVR or DL models to 540 obtain the values of the parameters of the model of the primary electron beam, $E_{P R E D}, S_{P R E D}$, 541 and $\alpha_{P R E D}$, shown in the fourth and fifth columns of Table 1, respectively. Because there was 542 no possibility to train a regressor for predicting the value of $\sigma_{E}, \sigma_{E}=0.50 \mathrm{MeV}$ was 543 consistently used throughout. These initial predictions were next fed as input to the 544 reconstruction-based minimization procedure. After optimizing these predicted values for 545 either model, usually identical (or very similar) sets of finally estimated parameter values: $546 E_{F I N A L}, S_{F I N A L}$, and $\alpha_{F I N A L}$, shown in column 5 of Table 1, were obtained. These finally 547 estimated electron beam model parameters values were then used to calculate the 548 reconstructed dose profiles. The measured and reconstructed profiles in each of the three 549 experimental cases are compared in Fig. 8. The mean values of absolute differences between 550 the measured and reconstructed profiles are given in the last column of Table 1. 
The first set of measured profiles (Case 1) consisted of five profiles: two depth

552 profiles and three lateral profiles, one of which was measured at the depth of $D_{\max }=1.4 \mathrm{~cm}$, as

553 listed in Table 1. The depth profiles were measured to a depth of $35 \mathrm{~cm}$ while the ranges of

554 measurement of lateral profiles were adjusted to the field size. The ranges of training data for

555 this set of profiles were adjusted to the ranges of real measurements prior to being applied to

556 train the PCA+SVR or DL models. The returned sets of values of the predicted parameters

557 from either model are listed in columns 4 and 5 of Table 1, for the PCA+SVR and DL models, respectively. As mentioned earlier, $\sigma_{E}=0.50 \mathrm{MeV}$ was consistently used. A reconstruction-

559 based minimization procedure was then applied to these predicted values, resulting in

560 identical values of final estimates, $E_{F I N A L}, s_{F I N A L}$, and $\alpha_{F I N A L}$ for either model, as listed in

561 column 6 of Table 1. The mean absolute differences between the measured profiles and

562 profiles reconstructed using these final parameter values (column 7 in Table 1) are shown in

563 the last column of Table 1. The measured and reconstructed profiles for this case are shown in

\section{Fig. 8a.}

The second set of measurement profiles (Case 2) consisted of one depth profile and

three lateral profiles, one of which was measured at depth $D_{\max }$, listed in column 2 of Table 1.

567 The depth profile was measured to a depth of $30 \mathrm{~cm}$ while the ranges of measurement of 568 lateral profiles were adjusted to the field size. The regression models were retrained for this

569 set of profiles and returned the initial guesses for the primary beam parameter values shown in 570 column 4 of Table 1. After optimization, the final prediction values shown in column 6 of 571 Table 1 were obtained. The deep (DL) model retrained for this set of profiles was then applied 572 to predict values of electron beam parameters using this set of measurement profiles. The 573 prediction results of the DL model are listed in column 5 of Table 1. After optimization, the 574 same set of final prediction values as those from the PCA+SVR model was obtained, i.e., 575 those shown in column 6 of Table 1. The mean absolute differences between the profiles: 
measured and reconstructed using these final beam parameters are shown in the last columns

577 of Table 1. The measured and reconstructed profiles for this case are shown in Fig. $\mathbf{8 b}$. Finally, the third set of measurement profiles (Case 3) consisted of six profiles: a

579 depth profile and two lateral profiles, both at $\mathrm{D}_{\max }$ depth. The depth profile was measured to a

580 depth of $30 \mathrm{~cm}$ while the ranges of measurement of lateral profiles were adjusted to the field

581 size. The regression models were retrained for this set of profiles and returned an initial guess

582 for the primary beam parameter values shown in column 4 of Table 1 . After optimization, the

583 final predictions for the PCA+SVR model are shown in column 6 of Table 1. The DL model

584 was also trained for this set of profiles and the values of predicted parameters of the primary

585 electron beam are listed in column 5 of Table 1. After repeated optimization of the same set,

586 again the set of finally predicted parameter values from the DL model and that from the

587 PCA+SVR optimized models, were identical (column 6 in Table 1) The mean absolute

588 differences between the profiles measured and those reconstructed using these last parameter

589 values are shown in the last column of Table 1. The measured and reconstructed profiles for

590 this case are shown in Fig. 8c.

591 Commenting generally on the results obtained, one should note the remarkably

592 consistent estimates of beam parameters obtained using either the PCA+SVR or the deep

593 learning models, and the excellent agreement between the reconstructed and measured

594 profiles, especially using the set of measured profiles and fields in the Case 3 study. However,

595 even in the Case 1 study, the somewhat higher discrepancies observed at the borders of the

596 lateral fields are to be expected. Over such regions of high dose gradients, higher uncertainties

597 may be due to measurement uncertainties, to averaging of input data by the phantom software 598 or to averaging of data in the training profiles, all affecting the quality of reconstructed 599 profiles over such regions. The excellent agreement between the reconstructed and real 
600 profiles is confirmed by the low values of mean absolute errors displayed in column 6 of

601 Table 1 in most cases ranging around $0.5 \%$ and exceeding $1 \%$ only once.

604 and their depth to be applied in simulator tuning should be guided by the expected range of

605 fields to be applied most frequently - hence, e.g., smaller fields should be selected if such

606 fields are expected to be applied more often in clinical work.

While there is a very good agreement between measured and profiles reconstructed

608 from regression results, based on PCA models, a final check of the proposed framework and

609 must be comparison of measured and simulated profiles. To this end, the dose delivery was

610 simulated for $10^{9}$ histories, using the virtual primary electron settings determined for Case 3

611 measurement experiment. Real profiles and simulated profiles for this case are compared in

612 Fig. 9.

\section{IV. Discussions}

615 Cancer radiotherapy using external photon beams, often termed conventional radiotherapy is

616 the most frequently applied form of radiotherapy world-wide. However, contemporary 617 elements of this type of radiotherapy - the linear accelerator, the therapy planning software 618 and quality assurance procedures which presently rely on up-to date technology and computer 619 engineering are far from being conventional. Among the critical issues in radiotherapy are the 620 accuracy and repeatability of dose delivery which requires that within the combined system of 621 a medical accelerator, therapy planning and clinical dosimetry, identical absolute dosimetry 622 units are exchanged. Thus, accurate quantitative description of the features of the model of the 623 primary electron beam in a linear accelerator is a major task of critical importance, being 624 immediately related not only to the accuracy of the therapy planning calculations but 
625 primarily to the accuracy of dose delivery to the patient - also affected by the correctness of

626 the relative calibration of the therapy planning system. For purposes of routine QA in the

627 clinic it is impossible to measure any parameters of the primary electron beam directly - the

628 only indirect option available is to estimate them on the basis of the dose distribution

629 measured in a clinical water phantom within a well-specified dosimetry procedure.

630 Development of a Monte Carlo-based model to relate specific features of the in-phantom

631 measured dose distributions with values of the basic parameters of the model of the electron

632 beam in a medical accelerator was the prime motivation in this work. Availability of an

633 elsewhere-developed complete MC model of the accelerator, i.e., of the PRIMO Monte Carlo

634 software, and development and successful application of statistical learning technology made

635 it possible to accomplish this task.

Ideally, for the solution of such a task to be of practical utility, it should be delivered

637 as a model which accepts at its input a well-defined assembly of measured profiles, returning

638 estimated values of the parameters characterizing the primary electron beam of a given

639 accelerator, together with dose profiles reconstructed basing on these estimated parameters.

640 Clearly, any discrepancy between the measured and the reconstructed profiles should be as

641 small as possible, which depends on the data used to train the learning models. Customization

642 of model inputs is a major obstacle, as medical physics departments often apply different

643 measurement tools, phantoms or measurement protocols. For these reasons, any specifically

644 defined procedure which requires measurements of a strictly limited and predefined set of

645 profiles according to a unique protocol would not be a solution likely to be universally 646 accepted.

The solution presented in this study is flexible and readily usable. Based on Monte

648 Carlo simulation data, a set of models was developed which extract features from a user-

649 defined collection of dose profiles to estimate primary electron beam model parameters from 
650 such features and returns reconstructed profiles for comparison with those measured and used 651 as input. Estimation of primary electron beam model parameters involves two steps - an 652 initial guess and optimization of the solution based on a model of the features of the measured 653 profiles. In contrast to all the work published so far, the characteristics of the dose profile 654 shapes and the regression functions are both machine-learned and collected in a data655 dependent manner. Neither hand-crafted shape features nor ad-hoc regression functions need 656 to be applied, these being replaced by a well-established background of statistical learning. 657 The two models developed in this work - one based on PCA feature extraction and SVR 658 regression and another, based on end-to-end deep-learning which simultaneously learns to 659 represent the shapes of the dose profiles and to apply the most suitable regression functions 660 are the proposed solution. Such a solution will support several different experimental 661 arrangements, offering optimum regression models for any such arrangement. By studying a 662 few experimental cases, the effect of the selection of the experimental setup on the accuracy 663 of parameter estimation has been demonstrated and discussed.

664 As was already mentioned, estimation of the primary electron beam model parameters 665 involves two steps, the first of which is an initial guess made by a regression model. In 666 principle, this initial guess could be made without any such model - merely by a brute force 667 search over all collected profiles for a set of profiles that best fit the analysed profiles. The 668 second stage of estimation, which is based on reconstruction-based minimization, requires 669 that techniques be developed to effectively represent the shape of the measured profiles - as 670 introduced in the present work. It should also be noted that a brute force search delivers no 671 explanatory power, in contrast to regression models introduced in the present study. In 672 particular, regression models deliver an association between explanatory and explaining 673 variables - for example, given a regression model it can be inferred in what manner will any 674 specific changes of primary electron beam model parameters influence the shapes of the 
675 resulting dose profiles. This is the general advantage of regression models over any brute

676 force search strategies, which is why regression models are widely used in statistical data 677 analysis.

678 The developed framework was tested using both simulated and real data. The tests

679 based on simulated data demonstrated that the coefficient of determination of true primary

680 beam parameters from dose profiles varies from around $92 \%$ for angular beam divergence to $68197 \%$ for mean energy of the simulated electron beam. It was not possible to train the 682 developed model to predict the FWHM of energy spectrum of primary electrons, implying 683 that this particular beam parameter does not seriously affect the shapes of dose profiles, at 684 least for the cases studied in this work.

685 The presented framework has been made freely available together with the simulation 686 data used for training the models. Model training and testing stages do not require extensive computation resources. Using any up-to-date PC with no graphic card support, the PCA+SVR models can be trained within a few seconds and prediction takes no longer than a second. The 689 training of deep learning models usually requires about ten hours of an average CPU. 690 However, testing the deep models takes no longer than testing the PCA+SVR model. The presented framework can be readily adapted to individual requirements, perhaps 692 guided by the availability of profile sets prepared for QA purposes, or by ease of 693 measurement. Dose data could also be supplied by dose distributions measured by detectors 694 other than ionization chambers, e.g., dye films, especially over regions of high dose gradient. 695 Indeed, for any selection of profiles which the user intends to apply in determining values of 696 parameters of the primary electron beam models, only a few lines of the configuration code 697 need to be changed to indicate such user-specified selection. Then, the regression models 698 must be retrained, which takes only a few seconds with no user intervention, except for 699 running the code. Following this training run, the estimation of electron beam model 
700 parameters and reconstruction of profiles from estimation results can be executed - this

701 requiring a few more seconds, provided that the measured doses are read by a script. Three

702 examples of such procedures for reading measured doses from text files have also been

703 provided in the freely available repository at https://github.com/taborzbislaw/DeepBeam .

\section{Conclusion}

706 The purpose of the present study was to develop a flexible framework with suitable regression

707 models for estimating parameters of the model of primary electron beam in simulators of

708 medical linear accelerators, basing on real reference dose profiles measured in a water 709 phantom. The proposed framework is a readily applicable and customizable tool which may

710 be applied in tuning virtual primary electron beams of Monte Carlo simulators of linear

711 accelerators. The codes, training and test data, together with some trained models and readout

712 procedures, are freely available at the site: https://github.com/taborzbislaw/DeepBeam.

\section{Abbreviations}

715 MC: Monte Carlo, EBT: external beam photon therapy, TPS: therapy planning system, QA:

716 quality assurance, FWHM: full width at half maximum, PCA: Principal Component Analysis,

717 SVR: Support Vector Regression, DL: deep learning

\section{Acknowledgements}

719 This research was supported by PLGrid Infrastructure. The authors wish to thank Mr. Lukasz

720 Flis of the ACC Cyfronet AGH for his invaluable help in handling computations on the

721 Prometheus grid.

\section{Author's contributions}


723 ZT conceived the work, conducted all the simulations, performed the subsequent analyses,

724 wrote the manuscript and revised it. DK conducted the measurements, wrote the manuscript

725 and revised it. MW wrote the manuscript and revised it.

\section{Funding}

727 This study was supported by the Foundation for Polish Science under grant POIR.04.04.00-

728 00-15E5/18.

\section{Availability of data and materials}

730 All data and code are available at https://github.com/taborzbislaw/DeepBeam.

\section{Ethics approval and consent to participate}

732 Not applicable.

\section{Consent for publication}

734 All authors consent to the publication.

\section{Competing interests}

736 The author declares that she has no competing interests.

\section{References}

739 [1] Storchi P and Woudstra E. Calculation of the absorbed dose distribution due to irregularly 740 shaped photon beams using pencil beam kernels derived from basic beam data. Phys Med 741 Biol. 1996;41(4):637-56

742 [2] Ulmer W, Pyyry J, Kaissl W. A 3D photon superposition/convolution algorithm and its 743 foundation on results of Monte Carlo calculations. Phys Med Biol. 2005;50(8):1767-90

744 [3] Failla GA, Wareing T, Archambault Y, Thompson S. Acuros XB advanced dose 745 calculation for the Eclipse treat-ment planning system. Palo Alto, CA: Varian Medical 746 Systems; 2010 
747 [4] Chetty IJ, Curran B, Cygler JE, et al. Report of the AAPM Task Group 105:

748 Issues associated with clinical implementation of Monte Carlo-based photon and

749 electron external beam treatment planning. Med Phys. 2007;34(12):4818-53

750 [5] Knöös T, Wieslander E, Cozzi L et al. Comparison of dose calculation algorithms for 751 treatment planning in external photon beam therapy for clinical situations. Phys Med Biol. $752 \quad 2006 ; 51(22):$ 5785-5807.

753 [6] Björk P, Knöös K, Nilsson P. Influence of initial electron beam characteristics on Monte 754 Carlo calculated absorbed dose distributions for linear accelerator electron beams. Phys Med 755 Biol. 2002; 47(22): 4019-4041.

756 [7] Tzedakis A, Damilakis JE, Mazonakis M, Stratakis J, Varveris H, Gourtsoyiannis N. 757 Influence of initial electron beam parameters on Monte Carlo calculated absorbed dose 758 distributions for radiotherapy photon beams. Med Phys. 2004; 31(4): 907-913.

759 [8] Maskani R, Tahmasebibirgani MJ, HoseiniGhahfarokhi M, Fatahiasl J. Determination of 760 Initial Beam Parameters of Varian 2100 CD LINAC for Various Therapeutic Electrons Using 761 PRIMO. Asian Pac J Cancer Prev. 2014; 16(17): 7795-7801.

762 [9] Pena J, Gonzalez-Castano DM, Gomez F, Sánchez-Doblado F, Hartmann GH. Automatic 763 determination of primary electron beam parameters in Monte Carlo simulation. Med Phys. 764 2007; 34(3): 1076-1084.

765 [10] Mayles P, Nahum A, Rosenwald JC, Eds. Handbook of Radiotherapy Physics. Boca 766 Raton: CRC Press; 2007.

767 [11] Jiang SB, Kapur A, Ma CM. Electron beam modelling and commissioning for Monte 768 Carlo treatment planning. Med Phys. 2000; 27(1): 180-191.

769 [12] Almberg SS , Frengen J, Kylling A, Lindmo T. Monte Carlo linear accelerator simulation 770 of megavoltage photon beams: independent determination of initial beam parameters. Med $771 \quad$ Phys. 2012; 39(1): 40-47. 
772 [13] Maskani R, Tahmasebibirgani MJ, HoseiniGhahfarokhi M, Fatahiasl J. Determination of 773 Initial Beam Parameters of Varian 2100 CD LINAC for Various Therapeutic Electrons Using 774 PRIMO. Asian Pac J Cancer Prev. 2014; 16(17): 7795-7801.

775 [14] Park H, Choi HJ , Kim JI, Min CH. Analysis of Dose Distribution According to the 776 Initial Electron Beam of the Linear Accelerator: A Monte Carlo Study. Journal of Radiation

777 Protection and Research. 2018; 43(1):10-19.

778 [15] Mohammed M, El Bardouni T, Chakir E, Boukhal H, Saeed M, Ahmed AA. Monte Carlo 779 simulation of Varian Linac for $6 \mathrm{MV}$ photon beam with BEAMnrc code. Radiation Physics 780 and Chemistry. 2018; 144 (March 2018): 69-75.

781 [16] Tugrul T, Erogul O. Determination of initial electron parameters by means of Monte 782 Carlo simulations for the Siemens Artiste Linac 6 MV photon beam. Reports of Practical 783 Oncology and Radiotherapy. 2019; 24(4): 331-337.

784 [17] Najafzadeh M, Hoseini-Ghafarokhi M, Mayn Bolagh RS, Haghparast M, Zarifi S, 785 Nickfarjam A, Farhood A, Chow JCL], J Xray Sci Technol 2019;27(6):1047-1070 786 Benchmarking of Monte Carlo model of Siemens Oncor ${ }^{\circledR}$ linear accelerator for 18MV photon 787 beam: Determination of initial electron beam parameters

788 [18] Bacala AM. Linac photon beam fine-tuning in PRIMO using the gamma-index analysis 789 toolkit. Radiat Oncol. 2020; 15: 8.

790 [19] Rodriguez M, Sempau J, Brualla L. PRIMO: a graphical environment for the Monte 791 Carlo simulation of Varian and Elekta linacs. Strahlentherapie und Onkologie. 2013; 792 189(10): 881-886.

793 [20] Salvat F, Fernández-Varea JM, Sempau J. PENELOPE 2011-a code system for Monte 794 Carlo simulation of electron and photon transport. Issy-les-Moulineaux: OECD Nuclear 795 Energy Agency; 2011. 
796 [21] Brualla L, Sauerwein W. On the efficiency of azimuthal and rotational splitting for 797 Monte Carlo simulation of clinical linear accelerators. Radiat Phys Chem. 2010; 79 (9): 929 $798 \quad 932$

799 [22] Jolliffe IT. Principal component analysis, New York: Springer; 2002.

800 [23] Vapnik VN. Statistical Learning Theory. New York: Wiley; 1998.

801 [24] Kraft D. A software package for sequential quadratic programming. Tech. Rep. DFVLR802 FB 88-28, Koln: DLR German Aerospace Center — Institute for Flight Mechanics; 1988.

803

804 


\section{Figure legends}

806 Fig. 1 Scheme of the deep learning regression model architecture.

807 Fig. 2 Scheme of the method of reconstructing profiles from regression results.

808 Fig. 3 (a) Fraction of explained variance in the shapes of profiles, versus number of PCA

809 features. The error bars represent standard deviation of the explained variance values,

810 calculated for six profiles of three squared fields $\left(3 \times 3 \mathrm{~cm}^{2}, 10 \times 10 \mathrm{~cm}^{2}\right.$ and $\left.30 \times 30 \mathrm{~cm}^{2}\right)$; (b)

811 Fraction of profile shape variance explained by the first three features, as averaged over the 812 three squared fields, for six profiles (depth profile: $\mathrm{ID}=1$, and five lateral profiles at depths

$813 \mathrm{D}_{\max }=1.4 \mathrm{~cm}, 5 \mathrm{~cm}, 10 \mathrm{~cm}, 20 \mathrm{~cm}$, and $30 \mathrm{~cm}$, IDs from 2 to 6 , respectively).

814 Fig. 4 Variation in profile shapes in relation to any one of the first three PCA features being

815 either negative or positive (left, middle and right panels) for a $10 \times 10 \mathrm{~cm}^{2}$, lateral profile at 30

$816 \mathrm{~cm}$ depth (upper panels) or for a depth profile of a $10 \times 10 \mathrm{~cm}^{2}$ field (lower panels). For 817 explanation of "mean shape", "negative feature" and "positive feature" labels, see text.

818 Fig. 5 Coefficient of determination between ground truth and predicted values of energy, spot

819 size, and angular divergence, for regression based on all of three fields $\left(3 \times 3 \mathrm{~cm}^{2}, 10 \times 10 \mathrm{~cm}^{2}\right.$

820 and $30 \times 30 \mathrm{~cm}^{2}$ ) and a different number of profiles of each field. For further details, see text.

821 Fig. 6 Testing results for the PCA+SVR model.

822 Fig. 7 Testing results for the deep learning (DL) model.

823 Fig. 8 Measured (real) depth and lateral profiles and respective profiles reconstructed using 824 PCA and the finally estimated beam parameters (listed in column 6 of Table 1), for three cases 825 of experimental design. The specification of profiles compared in each panel are listed in 826 columns 2 and 3 of Table 1: (a) Case 1; (b) Case 2; (c) Case 3.

827 Fig. 9 Measured (real) depth and lateral profiles and respective simulated profiles. The 828 simulation was run for $10^{9}$ histories for experimental settings and virtual primary electron 829 beam profile corresponding to Case 3. 


\section{Figures}
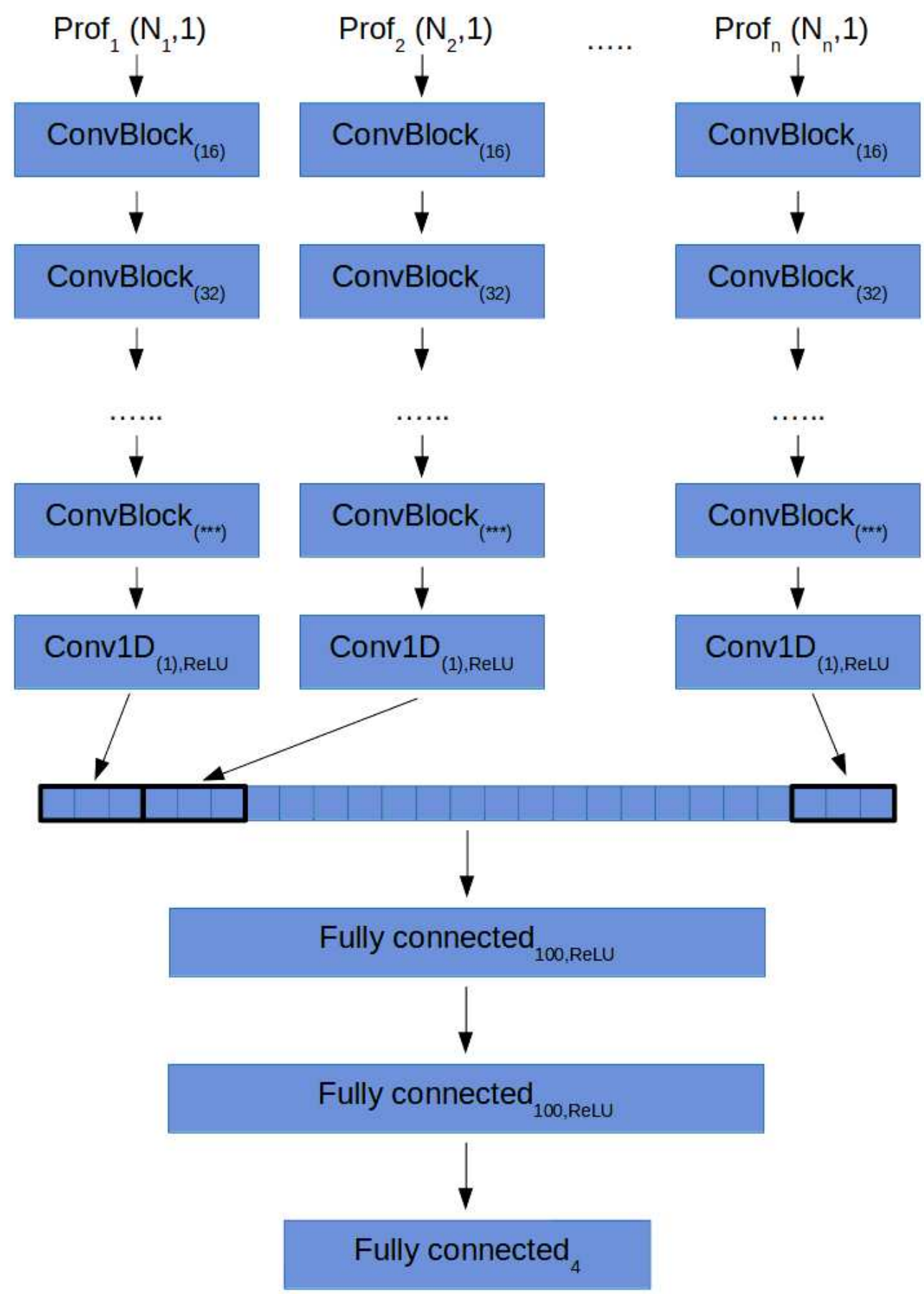

Figure 1

Scheme of the deep learning regression model architecture. 


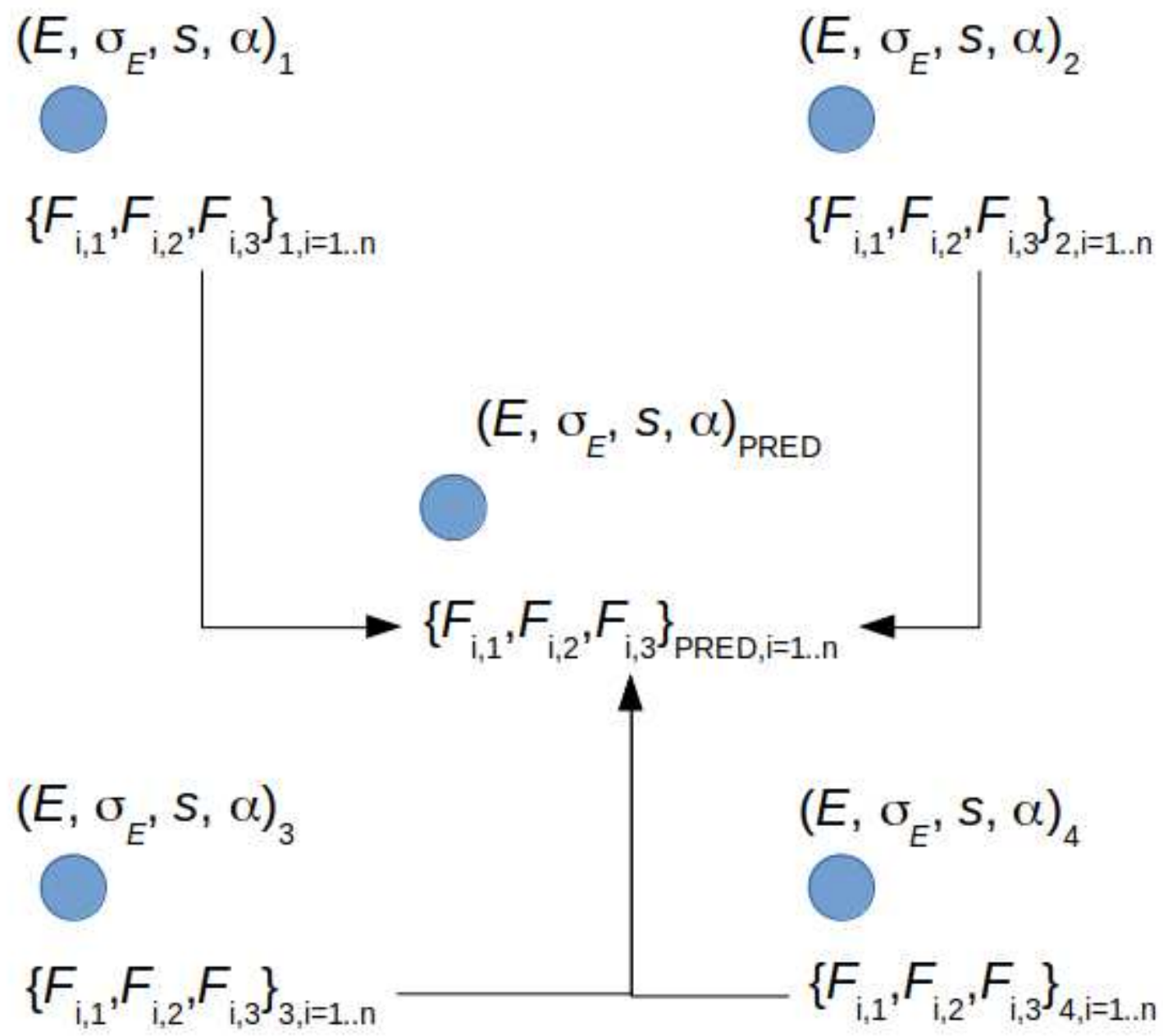

Figure 2

Scheme of the method of reconstructing profiles from regression results. 


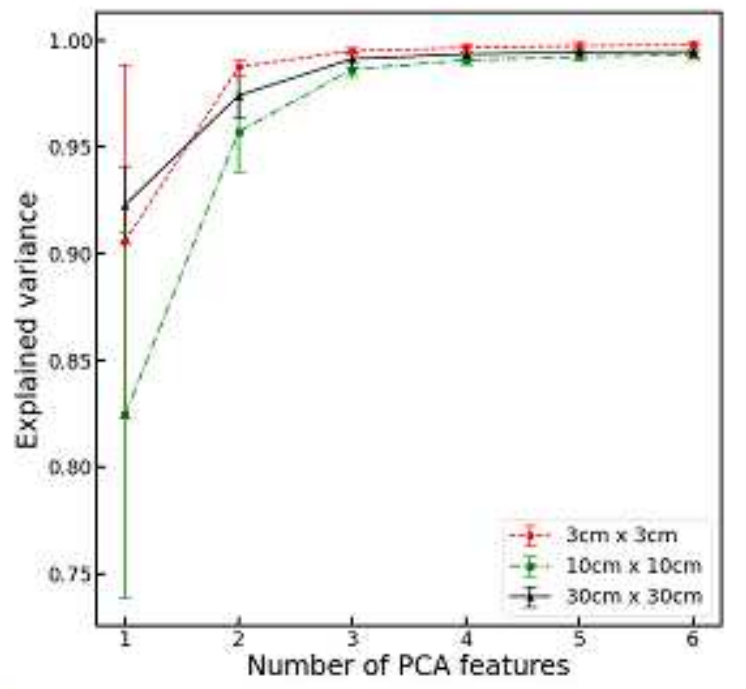

(a)

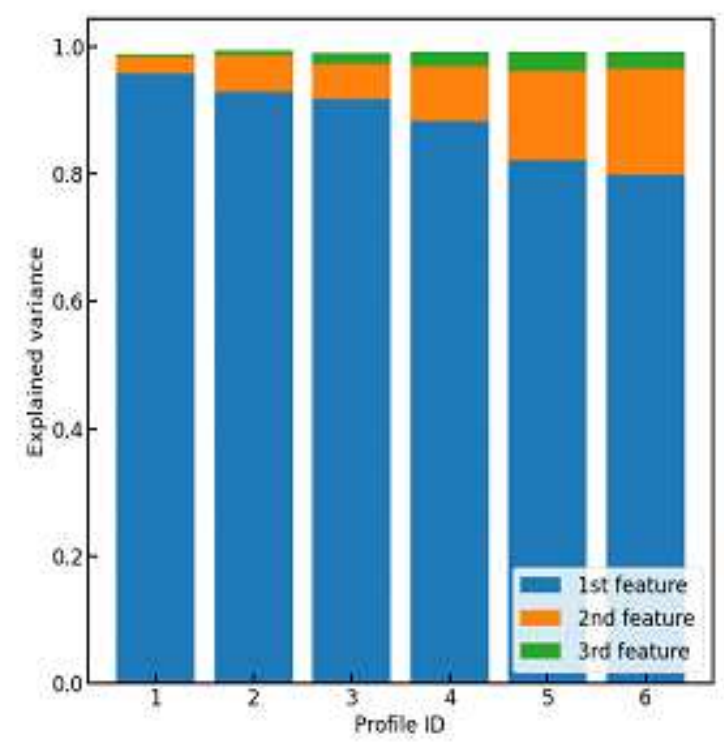

(b)

\section{Figure 3}

(a) Fraction of explained variance in the shapes of profiles, versus number of PCA features. The error bars represent standard deviation of the explained variance values, calculated for six profiles of three squared fields $(3 \times 3 \mathrm{~cm} 2,10 \times 10 \mathrm{~cm} 2$ and $30 \times 30 \mathrm{~cm} 2)$; (b) Fraction of profile shape variance explained by the first three features, as averaged over the three squared fields, for six profiles (depth profile: $I D=1$, and five lateral profiles at depths Dmax $=1.4 \mathrm{~cm}, 5 \mathrm{~cm}, 10 \mathrm{~cm}, 20 \mathrm{~cm}$, and $30 \mathrm{~cm}$, IDs from 2 to 6 , respectively). 

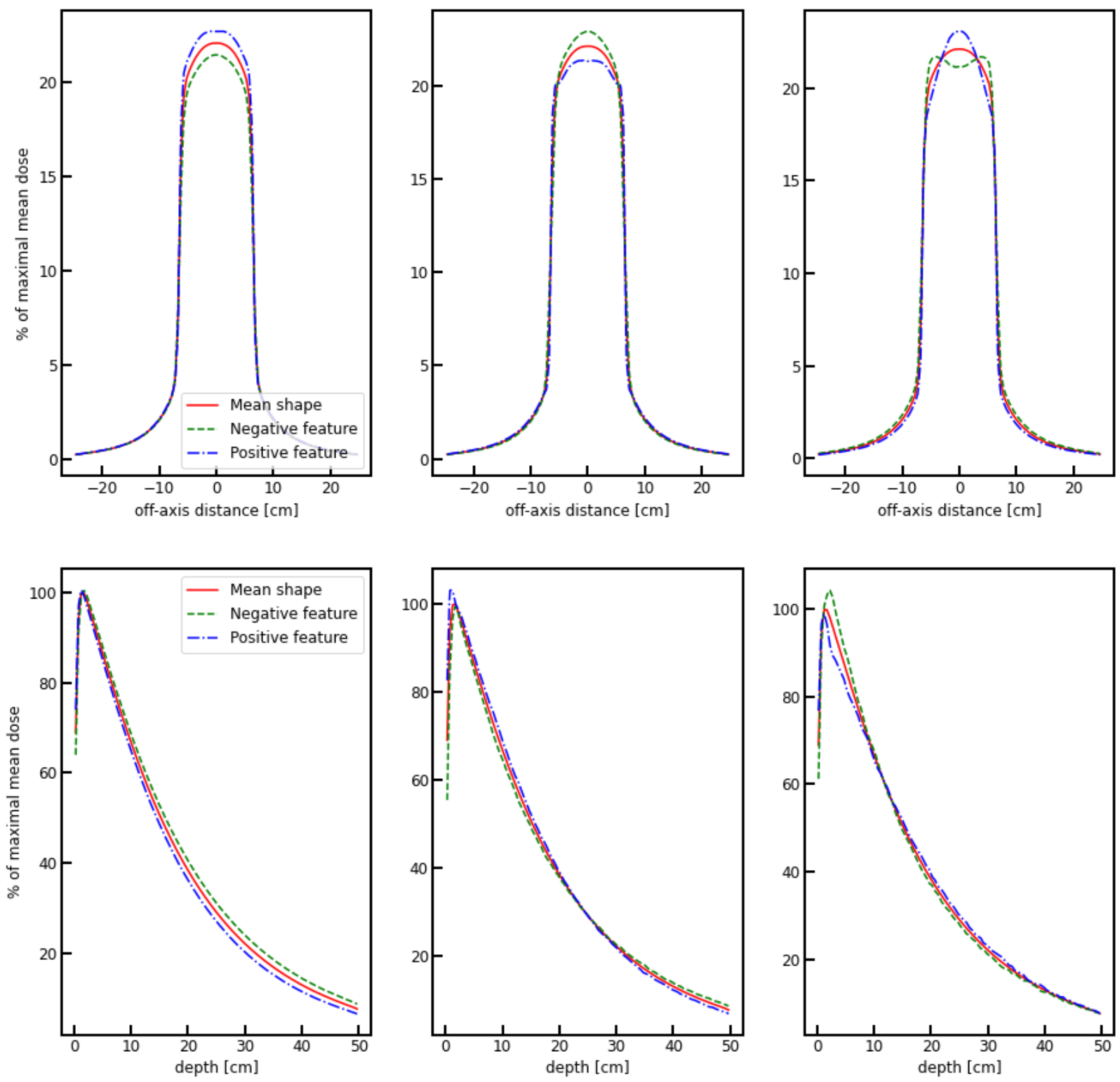

\section{Figure 4}

Variation in profile shapes in relation to any one of the first three PCA features being either negative or positive (left, middle and right panels) for a $10 \times 10 \mathrm{~cm} 2$, lateral profile at $30 \mathrm{~cm}$ depth (upper panels) or for a depth profile of a 10x10 cm2 field (lower panels). For explanation of "mean shape", "negative feature" and "positive feature" labels, see text. 


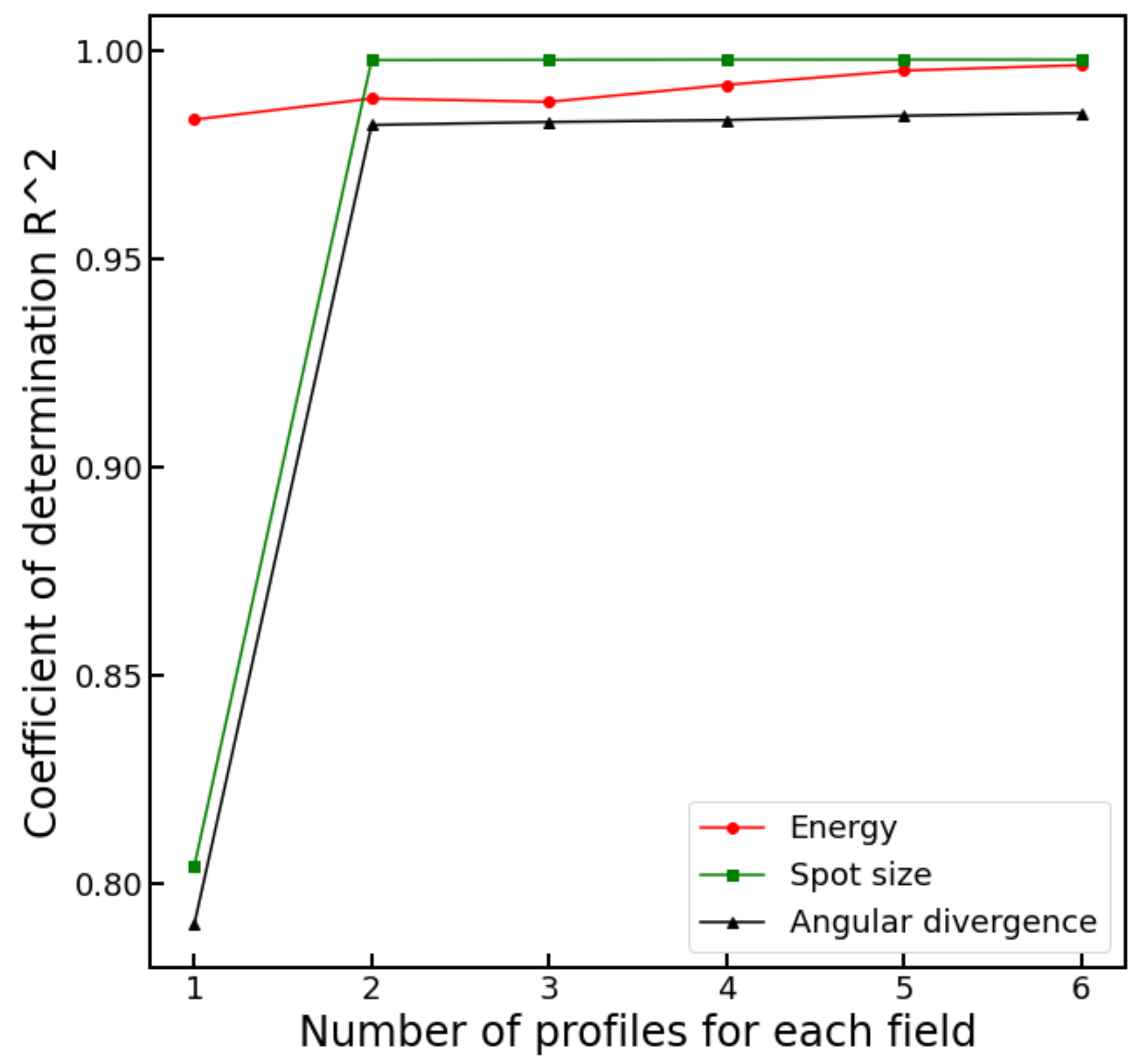

Figure 5

Coefficient of determination between ground truth and predicted values of energy, spot size, and angular divergence, for regression based on all of three fields $(3 \times 3 \mathrm{~cm} 2,10 \times 10 \mathrm{~cm} 2$ and $30 \times 30 \mathrm{~cm} 2)$ and a different number of profiles of each field. For further details, see text. 

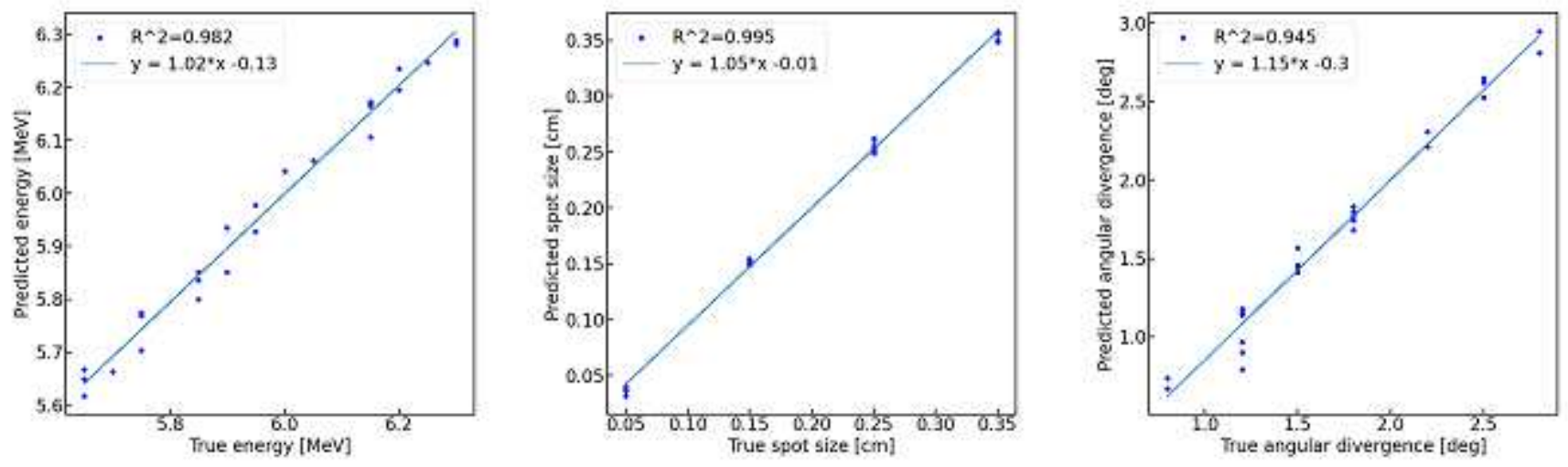

Figure 6

Testing results for the PCA+SVR model.
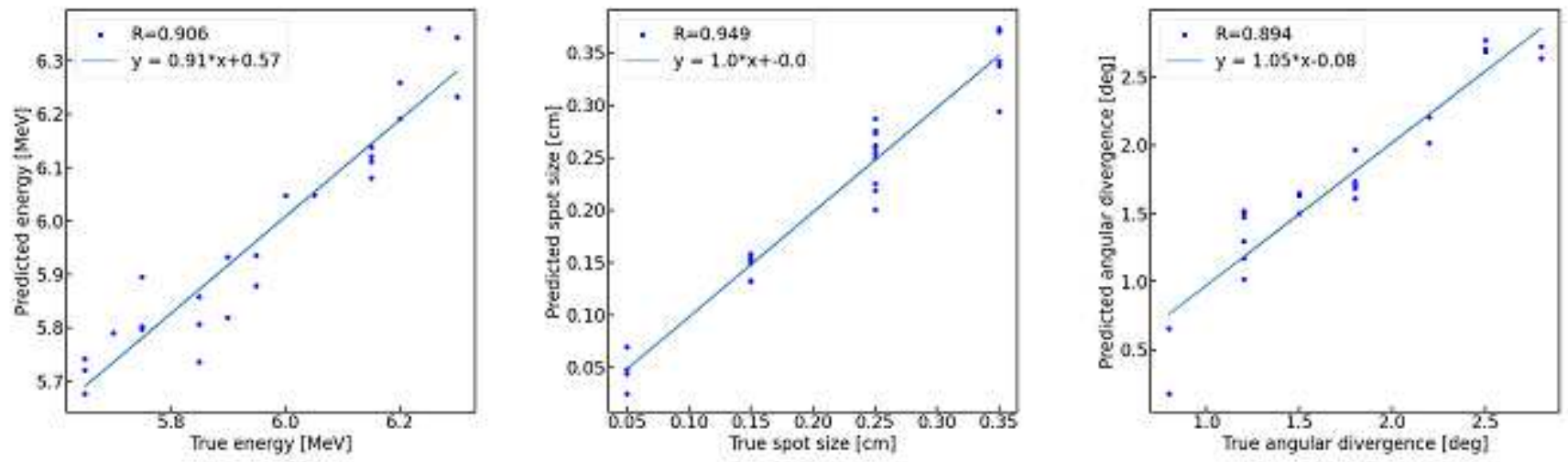

Figure 7

Testing results for the deep learning (DL) model. 

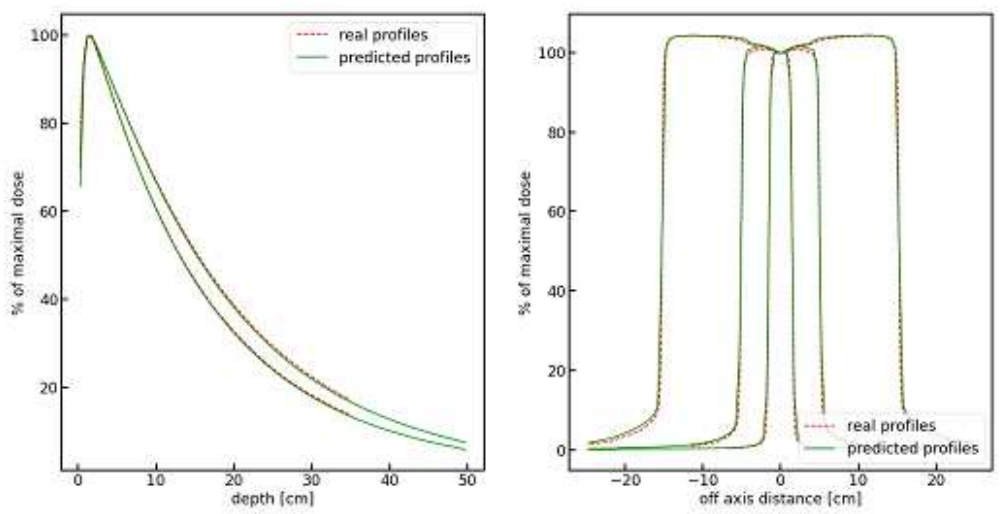

(a)
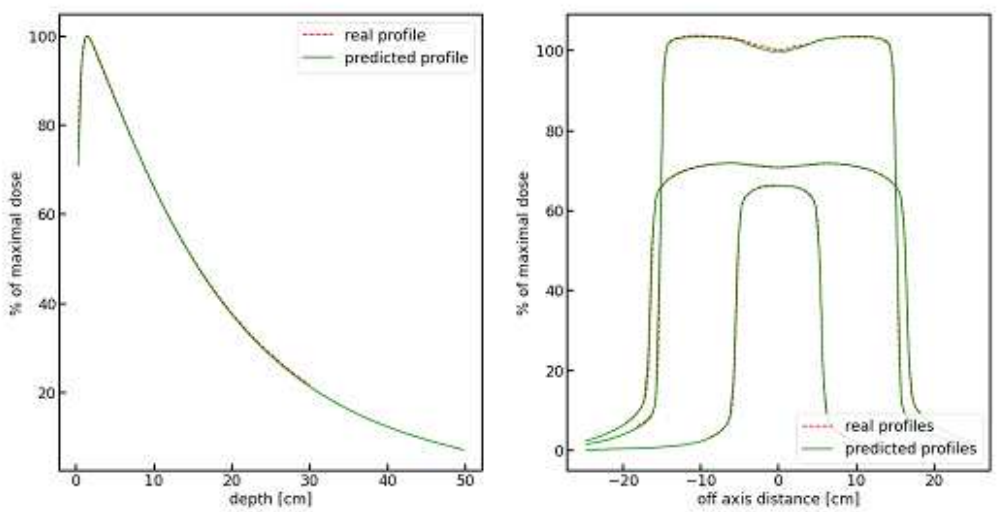

(b)
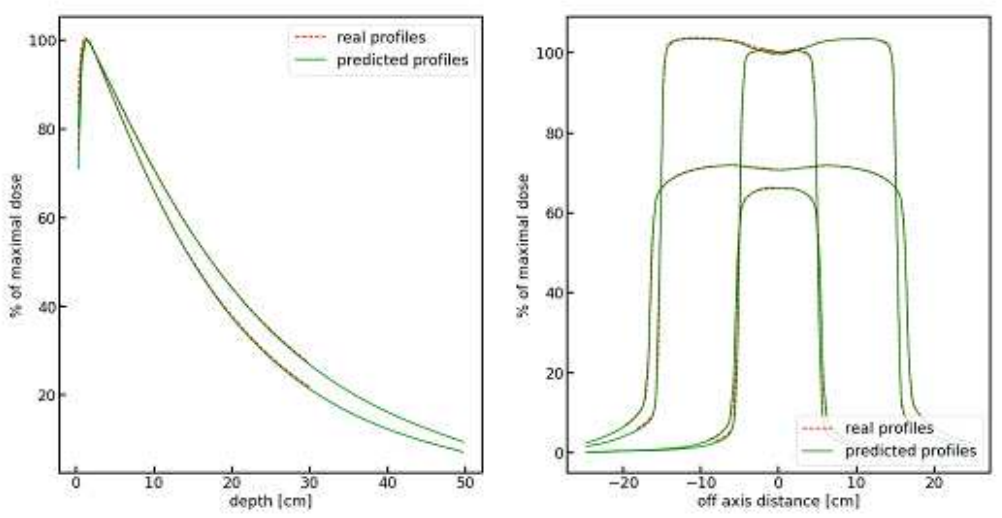

(c)

\section{Figure 8}

Measured (real) depth and lateral profiles and respective profiles reconstructed using PCA and the finally estimated beam parameters (listed in column 6 of Table 1), for three cases of experimental design. The specification of profiles compared in each panel are listed in columns 2 and 3 of Table 1: (a) Case 1; (b) Case 2; (c) Case 3. 

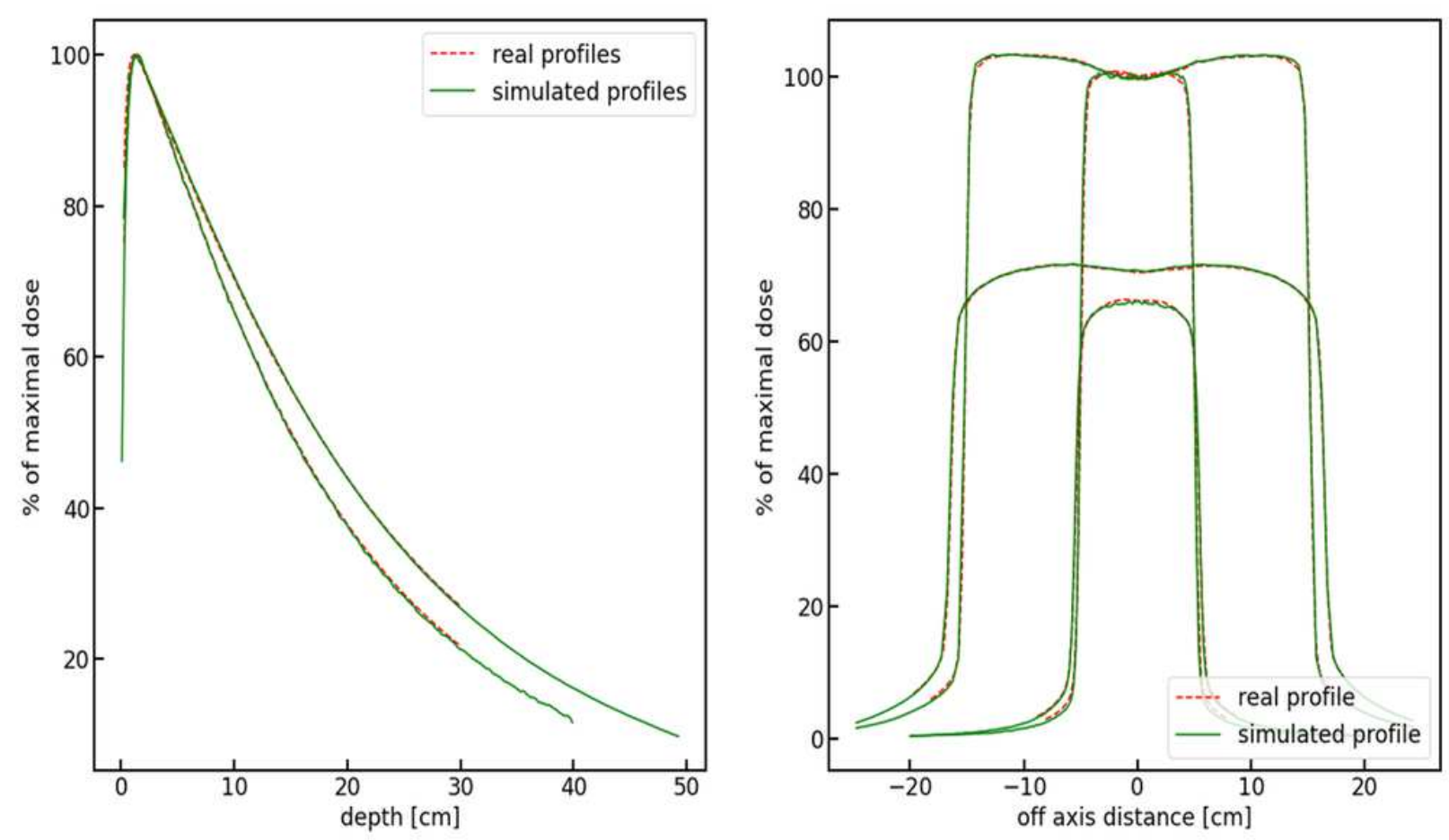

Figure 9

Measured (real) depth and lateral profiles and respective simulated profiles. The simulation was run for 109 histories for experimental settings and virtual primary electron beam profile corresponding to Case 3 . 